\title{
Spaz! The Effects of Local Latency on Player Actions in a Desktop-based Exergame
}

\author{
Wouter Durnez (D), Aleksandra Zheleva (D), Mark Claypool (D), Mathias Maes (D), Klaas Bombeke (D), \\ Jan Van Looy (iD, Lieven De Marez (iD)
}

\begin{abstract}
Technological advancement has decreased network latencies while simultaneously increasing local latencies. This may impact exergames-video games that incorporate exercisethe most since exergames tend to have complicated platforms to capture player actions. This paper presents a study using a custom desktop-based exergame that controls for local latency and measures player performance and Quality of Experience (QoE). Analysis of the results from a 37-person user study shows that while player performance and quality of experience degrades with latency, exergame actions are fairly tolerant of even hundreds of milliseconds of latency. Our data point towards a crucial tipping point at latency values of approximately 400 milliseconds.
\end{abstract}

Index Terms-latency, performance, quality of experience, exergaming, threshold

\section{INTRODUCTION}

$\mathbf{C}$ OMPUTER games are becoming a tool for improving health behaviors such as beneficial lifestyle habits and selfmanagement of illness and chronic conditions [1]. Exergamescomputer games that incorporate exercise into the game-have the potential to promote health by requiring physical activity in players as they play [2-4]. In addition to entertaining and helping socialization, exergames also have benefits to weight management, cardiovascular and physiological conditioning, and improving cognition [5].

However, imagine playing tennis where your body only responds to commands from your brain after a noticeable delay. Apart from ruining your chances of scoring a golden set, this handicap is also bound to be frustrating and ruin your fun. For computer game players, this phenomenon is known as lag or latency - that is, the temporal delay between player input and its perceived effect. For interactive applications, latency has two main sources. First, the computer platform on which the application is executed requires time to process the input and generate appropriate-usually visual-results. This is called input lag or local latency. Second, if the application needs to send data over a network before displaying results, this adds additional delay, called network latency.

Whereas local latency was often seen as a mere shortcoming in the computer's processing power, network latency was viewed as more difficult to mitigate. Consequently, a significant portion of prior research has focused on the relationship

Wouter Durnez, Aleksandra Zheleva, Klaas Bombeke, Jan Van Looy, and Lieven De Marez are with imec-mict-UGent, affiliated with imec and Ghent University, Ghent, Belgium.

Mark Claypool is with the Computer Science Department in Worcester Polytechnic Institute, MA, USA between network latency and measures of user-related outcomes, such as player performance [6] and the overall player experience [7]. In recent years, technological advancement has seen to a steady decrease in network latencies, in part due to increased bandwidth as reflected by Nielsen's law stating that a high-end user's network connection grows in capacity by $50 \%$ per year [8]. Local latency, conversely, may in fact be increasing [9] as wireless peripherals, high-latency displays, and next-generation input devices add more software layers and put more strain on local computer platforms.

Many exergames rely on motion-sensing interfaces that are specifically developed to quantify physical movement as well as maintain the game world. This often strains local computing platforms, yielding comparatively high local latencies. For example, the average local latency of the Sony PlayStation Move was 115 milliseconds, while the average local latency of the first generation Microsoft Kinect was 218 milliseconds [10]. Local latencies as low as 41 milliseconds have been shown to negatively affect performance in $2 \mathrm{D}$ mouseand-keyboard tasks [9]. This raises the research question: how does local latency impact player performance and experience in exergaming?

Prior research on the effects of latency on games has largely dealt with commercial entertainment games [11-15], which typically use mice and keyboards or game controllers and do not require the physical movements by players as do exergames. In addition, prior research on the effects of latency on game actions [16, 17] and user input [18-23] has primarily dealt with a traditional input device, such as a mouse and keyboard, and has not incorporated motion-sensing input devices.

While it is inviting to assume that the effects of latency on motion-sensing exergames can be derived from parallel research on entertainment games played with traditional controllers, such an approach would disregard the limitations of the human visual system. Specifically, it is more demanding for a person moving (e.g., exercising) to process visual input-an aptitude referred to as static-object dynamic visual acuity [24]. This may in turn interfere with a user's ability to detect visual aberrations, such as latency, potentially curtailing the impact of latency on quality of the experience and user performance.

The aim of the present study is to analyze the effects of local latency on an actively moving user in the context of an exergame. To this end, we conducted an experiment in which participants played a custom-made exergame called Spaz! using the Microsoft Kinect motion-sensing input device for the Xbox One. Players grabbed moving targets with their hands as fast as they could using an onscreen projection of their own silhouette 
produced by the Kinect. The game added different amounts of artificial latency at different sections of the game. Player performance was measured by recording reaction time-the time to see the target and select it by covering it with a hand silhouette. Quality of Experience (QoE), in turn, was measured using the 'flow' construct. First coined by Csikszentmihalyi in 1975 [25], flow can be understood as "a psychological state in which the person feels simultaneously cognitively efficient, motivated, and happy" [26]. Flow is often used to measure a player's experience while playing video games [27], and the 'flow' construct has been used as a quality measure in exergaming, as well [28].

The rest of this paper is organized as follows: Section II describes related work on exergames, latency and games, local latency, and measuring player experience; Section III details our methodology to measure the effects of latency on an exergame action; Section IV analyzes the results from our 37 person user study; Section V discusses the implications of our methods and findings; and Section VI summarizes our conclusions.

\section{RELATED WORK}

\section{A. Exergames}

An exergame is a computer game that also provides the game player with some physical activity. As such, exergames have the potential to subvert a sedentary lifestyle of playing computer games with one involving physical activity. Since the popularity boost given to exergames by the Nintendo Wii console, exergames have been expanding to other consoles (e.g., Microsoft xBox) as well as other platforms (e.g., smartphones), and have evolved into different subgenres: a) location based games that require players to traverse the real world; b) dancing games that require players to match onscreen prompts as they dance, and c) running games that require players to follow the game's instructions while running [29].

Prior research studies have supported the idea that playing exergames can promote good health. Wang and Perry [2] showed an increase in heart rate, oxygen rate and respiratory rate in 7-10 year olds that were playing exergames. However, since the observed changes were less than the national recommendations and also less than the changes observed during regular exercise, they concluded the exergames could not substitute for regular exercise. Kretschmann [3] measured physiological benefits of an exergame on college studentstheir measurements of average energy expenditure for some Nintendo Wii games showed comparable $\mathrm{kcal} / \mathrm{hour}$ to that of bicycling and swimming laps [4].

Our work builds upon the motivating exergame work by studying a fundamental exergame action for console-based exergames-moving a limb to a specific destination in response to an on-screen prompt.

\section{B. Effects of Latency}

Broadly, there two different approaches to research in understanding the impact of latency on computer games: topdown and bottom up. a) Top-down: Studies using specific games are a top-down approach, extending knowledge of latency and games one game at a time. Researchers have attempted to generalize the effects of latency to game genres (e.g., first person shooters) [11$15,30-32]$, typically by using a commercial game system and adding controlled amounts of latency as part of a user study. However, game design and game engines may obfuscate important system details [33], making it difficult for measured results to pertain to other games and to analytic modeling. Moreover, most prior top-down studies have dealt with online computer games where user input may be delayed by the roundtrip time to the server, unlike exergames where all latency may be local to the player's system only.

b) Bottom-up: An alternate approach is bottom-up, studying fundamental game input (e.g., target selection), refined (e.g., moving target selection) and differentiated by hardware (e.g., mouse). Research contributions to user input and latency has the potential to generalize to many games and even other interactive applications and allows for building analytic models that can explain and predict the effects of delay for a wide-range of games and delay conditions. Foundational studies of user input [18-22, 34] have shown promise in modeling user interaction for computer systems, including PC games [16], console games [35] and VR games [36]. However, such studies have not focused on exergame actions (e.g., full-body movement for target selection) nor have they focused on local latencies. Ideally, exergame designers and system developers would have a better understanding and perhaps models for the time it takes for an exergame player to select a moving target in order to better design the gaming experience.

For latency and full-body avatars, Waltemate et al. [37] study the effects of latency on users in a virtual CAVE environment, measuring motor performance and perception. They show latency above $75 \mathrm{~ms}$ can affect performance, albeit only slightly, and some measures of perception (agency and ownership) are resilient to much higher latencies. Participants' perceptions of latency are inferred by the task errors, suggesting latency perception may depend more upon performance than the actual delay. Caserman et al. [38] analyze the effects of latency in an immersive virtual reality environment, measuring the effects of latency on user performance in a reaching task and user perception in an embodiment task. They find latencies above $69 \mathrm{~ms}$ affect the time to reach an object, but agency or presence is resilient up to $250 \mathrm{~ms}$. Our results provide complementary measurements to this environment, where we find a tipping point to performance and user experience at higher latencies.

\section{Measuring player experience}

The construct of 'flow' is often used to measure a player's experience while playing video games [27]. First coined by Csikszentmihalyi in 1975 [25], flow can be understood as "a psychological state in which the person feels simultaneously cognitively efficient, motivated, and happy" [26]. Apart from its use in the evaluation of 'tradition' computer games [39, 40], flow has also been used as a quality measure in exergaming [28, $41,42]$. A similar concept exists in sports psychology, where flow is referred to as 'the zone' [43] - a state in which sports 
players are extremely focused and are performing at the peak of their potential.

In Csikszentmihalyi's framework, flow experiences are described as the composition of several elements, all of which contribute towards an engaging, joyful and optimal experience [44]. One of these components is 'agency', or the sense of control over one's actions. Previous research has shown that increasing amounts of latency can be harmful to a user's sense of agency [45]. As such, we expect our latency manipulation will have a measurable negative impact on the players' self-reported flow state.

\section{METHOD}

\section{A. Participants}

Thirty-seven people participated in this study, of which twenty-three were female. Their mean age was 28.19 years (SD 6.09). Twenty-eight participants indicated they had sometimes played video games on a daily basis, suggesting a degree of familiarity with gaming. Only three participants reported to have never played video games. Almost all participants were physically active to some degree in daily life (activity levels [46] were 16 high, 19 moderate, 2 low).

\section{B. Stimulus material and apparatus}

For the purpose of this experiment, a custom-made exergame ('Spaz!') was programmed using Unity (fig. 1). In the game, players saw their silhouettes on a television screen (a Samsung UE55D6750) that moved in unison with their own actions, save with some degree of - -experimentally induced-- latency. The screen was positioned $1.8 \mathrm{~m}$ in front of the participants. The silhouette was captured by a Microsoft Kinect for Xbox One, which was positioned behind the players. Players were asked to stay on a line that was taped to the floor, parallel to the screen, so their onscreen silhouettes would remain approximately the same size for the duration of the game.

The goal of the game was to 'grab' moving targets by covering the target with the hand of the silhouette. Targets moved with constant velocity away from the player initially, and then continued straight, bouncing off of the edges of the play field. Per trial, this velocity was randomly sampled from a uniform distribution, with lower bound $0.128 \mathrm{~m} / \mathrm{s}$ and upper bound $0.642 \mathrm{~m} / \mathrm{s}^{1}$. These boundaries were determined while piloting the game with no added latency in an effort to create a balanced game with a sufficiently diverse difficulty range. The target only disappeared after it was covered by either the silhouette's hands for 500 continuous milliseconds to encourage participants to be accurate and steady. After a successful 'grab', participants were required to reset to the middle of the play area with their hands next to their bodies before the next trial would commence.

For each trial, the game recorded the reaction time as the time between when the target appeared and the participant successfully grabbed it, minus 500 milliseconds (the time a

\footnotetext{
${ }^{1}$ These values are calculated using this formula: real target speed $=$ $1 / 5 \times$ game target speed $\times$ player distance to screen $\times \tan \left(35.3^{\circ}\right)$, where game target speed is measured in Unity units, and real target speed and player distance to screen in meters.
}

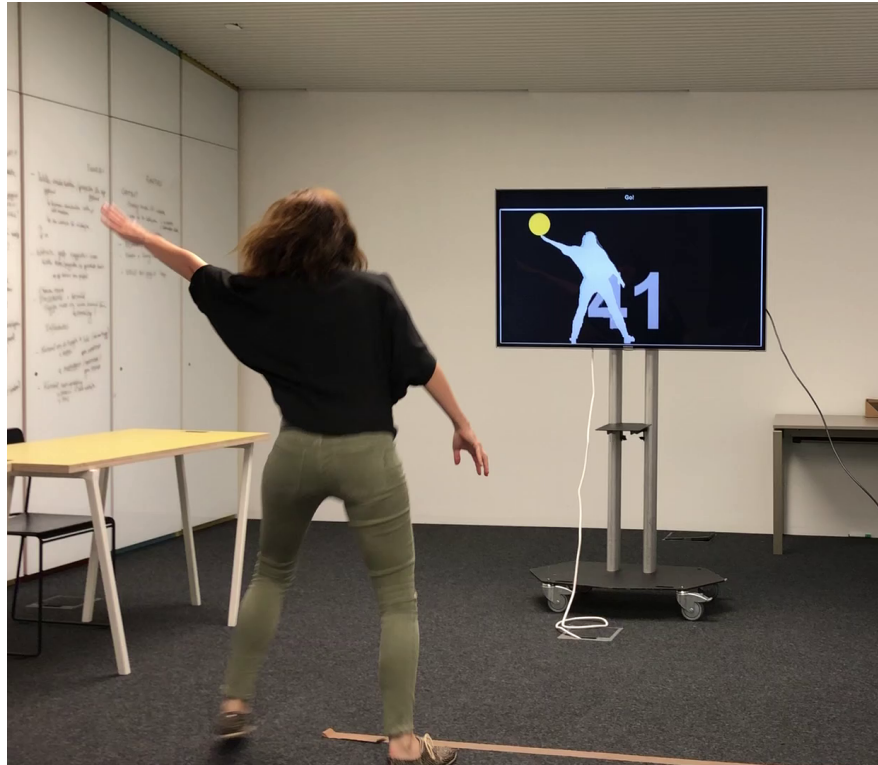

Fig. 1. Participant playing 'Spaz!'.

target had to be 'held' for it to disappear), minus the trial latency (see below). The game encouraged players to grab targets as quickly as possible by gradually changing the color of the target to indicate how many points could still be gathered by successfully grabbing it - that is, fast responses yielded more points. The score was shown on screen, behind the silhouette.

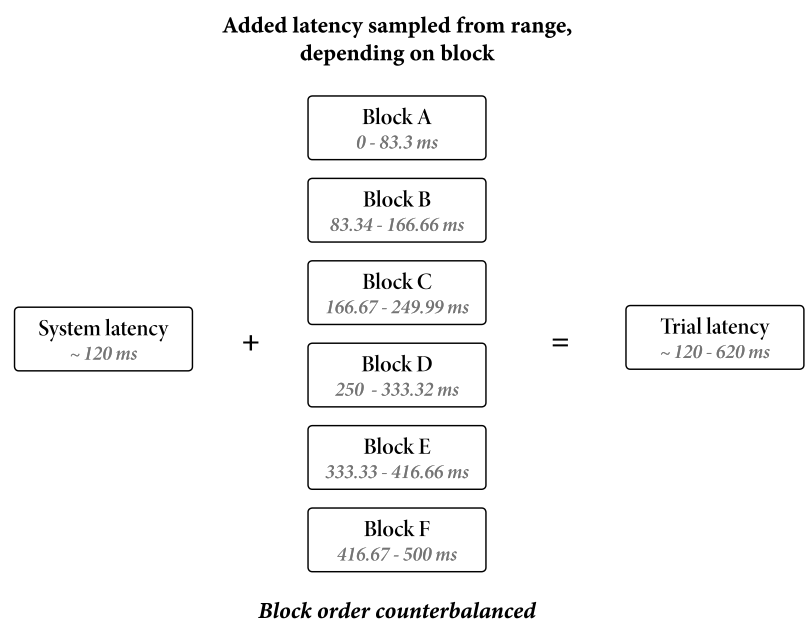

Fig. 2. Experiment design.

The game was divided into one practice block (10 trials) and six experiment blocks (40 trials each). In the practice block, no artificial latency was added. In each experiment block, a random amount of artificial latency was added to each trial, sampled from a uniform distribution defined separately for each (fig. 2). These ranges were determined based on a pilot run of the experiment. The order of the experiment blocks was counterbalanced.

After each experiment block, participants were asked to 
fill in a short survey. First, the survey gauged for latency awareness ("Indicate how large the delay was between what you did and what you saw."), sense of adaptability ("Indicate to what degree you could adapt to the delay."), sense of efficiency ("Indicate how efficiently you could play the game."), and disturbance ("Indicate how disturbing you found the latency."). These items have been used in a study on latency before [47]. In the present study, however, these items were rated using an anchored visual analogue scale. This approach does away with some of the typical problems with labeled Likert scales [48], providing linear data instead [49, 50]. Anchors were taken from the original items. Next, we measured flow, for which we used the Flow Short scale [51]. The last three items on the scale (measuring perceived importance) were dropped, leaving 10 items. Each item was scored on a visual analogue scale, retaining the original scale's anchors. Finally, we measured perceived competence and interest and enjoyment using the corresponding subscales of the Intrinsic Motivation Inventory (IMI) [52]. Again, responses were gathered using an anchored visual analogue scale response format. For statistical analysis and visualization, ratings and subscale sum scores were normalized (i.e., transformed to values between 0 and 1).

\section{Local Latency Measurements}

Ivkovic and colleagues measured local latency in a sample of PCs and gaming consoles connected to various displays [9]. Using an external high frame rate camera (240 f/s), they film from when input is applied (e.g., the mouse is moved) until the result is rendered on the display (e.g., the player's first-person view changes). By manually examining the individual video frames from input to result, they compute the local latency (in this case, with a granularity of 4 milliseconds). They found a wide range of latencies from $23 \mathrm{~ms}$ for a commercial firstperson shooter game on a PC with an ultra-fast gaming monitor, up to $243 \mathrm{~ms}$ for a popular third-person action game on a game console with a flat-screen television.

In order to overcome some of the potential shortcomings of using an external camera (e.g., frame rate limitations and detecting the input start time), Raaen and Petlund [53] propose connecting an oscilloscope to a computer mouse and the computer's display, thus providing a more precise elapsed time between the photosensor detecting the input from the mouse and the photosensor detecting pixel changes on the display. Preliminary results for a commercial first-person shooter game on a laptop show local latencies from $21 \mathrm{~ms}$ to $102 \mathrm{~ms}$.

Casiez et al. [54] present an alternate methodology for measuring local latency that consists of positioning an unmodified optical mouse on a screen while displaying a particular texture to fake mouse movement. Measurements across 10 mice, 2 displays, 2 operating systems and several applications and toolkits show local latencies vary from 46 to 83 milliseconds. These measurements do not include any additional processing that would typically occur in a game, such as processing by the game engine.

Raaen and Kjellmo [55] adapt the system to connect a laser pointer and a tripod-mounted camera to the oscilloscope, measuring the latency in popular VR devices-the Oculus Rift and several smartphones. They find a wide range of latencies, with the Oculus Rift platform yielding much lower latencies than smartphone platforms (Samsung Galaxy S4 \& S5, and iPhone 5s \& 6).

While providing methodologies to measure local latency and showing significant local lag for many typical computer setups, none of the above studies measured local latencies for a motion sensing input device, such as the Microsoft Kinect.

In our study, we measured our system's base latency as follows. Using Unity, we visualized a rectangle on the display. The Kinect camera was then used to detect a color change in this rectangle (from white to red). System base latency was calculated as the time between the color change and the registration through the Kinect. This process was repeated five times in rapid sequence and the mean used as the base latency. Since system latency was measured prior to each block to monitor the emergence of potential hidden system processes, this could inadvertently give rise to a deviation in the system latency. Over all experiment blocks and sessions, system latency averaged 121.38 milliseconds (SD 7.86), which is considerably higher than most traditional gaming setups. Depending on the experiment block, the experienced trial latency in our study could thus vary between approximately $121 \mathrm{~ms}$ (system base latency) and $620 \mathrm{~ms}$ (system base latency plus maximal artificial latency) (fig. 2).

\section{Procedure}

Participants were given an explanation of the goal of the game but were not informed of the goal of the experiment until after the game session. After hearing the game goals, participants filled in an informed consent form, as well as a short sociodemographic survey. Next, participants were made familiar with the game through a series of 10 practice trials. Then, participants played 6 experiment blocks, each followed by the post-block questionnaire. Finally, we explained the goal of the experiment to the participants and each was given a small remuneration for their time.

\section{ANALYSIS AND RESUlTS}

\section{A. Performance}

We analyzed player performance by modelling the impact of latency on players' reaction times. Aggregated statistics are given in table II. Figure 3 depicts a cumulative distribution graph of the reaction times for all users, clustered by latency block (the minimum added latency for that trial). The $\mathrm{x}$-axis is the user reaction time (in milliseconds) and the y-axis is the cumulative distribution. About $5 \%$ of the reaction time values are off the graph to the right. From the graph, the reaction times are heavily skewed, having maximum reaction times far above the median. Maximum reaction times (not shown since they are above the $\mathrm{x}$-axis range) are about 20 seconds for blocks A, B, and C and about 30 seconds for blocks D, E and F. The overall maximum reaction time is nearly 100 seconds. Note that these extreme values were a rare occurrence, caused by participants temporarily 'giving up' due to frustration.

We mitigated this skewness problem by fitting the reaction time data with quantile regression models, using the conditional 


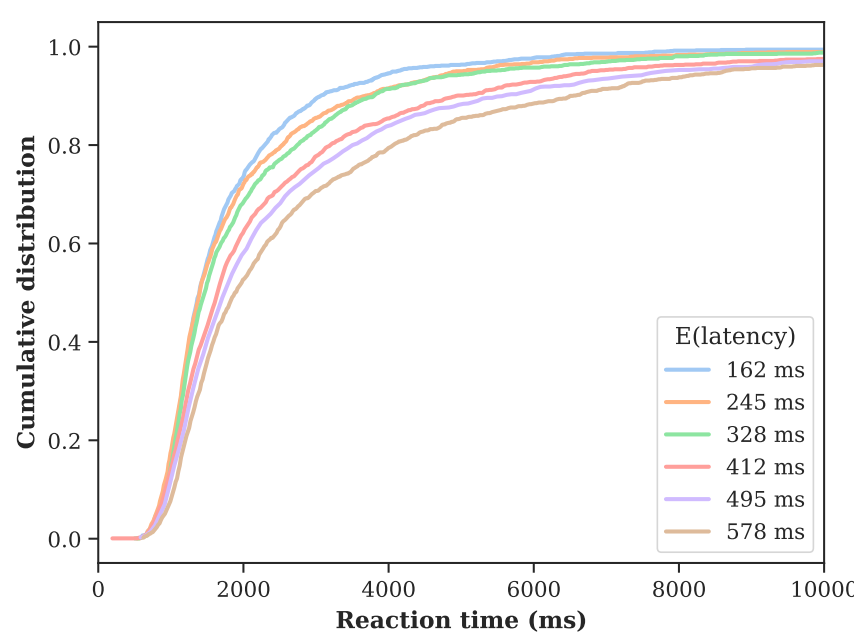

Fig. 3. Cumulative distribution of reaction time data for each latency block (minimum added latency).

median $(\tau=0.5)$. Quantile regression modeling does not make assumptions about the distribution of the residuals [56], making the technique especially suited to deal with this type of data. Specifically, our approach consisted of the following steps. First, we fit the reaction time data with a quantile regression model containing a single first order predictor: latency. We then introduced additional polynomial terms (up to the $4^{t} h$ degree) in an attempt to uncover non-linear relationships. Subsequent models were compared using F-tests: only those models that showed a statistically significant improvement to the previous best model were retained.

The model showing the best fit was expressed by a cubic polynomial (i.e., reaction time $\sim$ latency + latency ${ }^{2}+$ latency $^{3}$; see table I and fig. 4). Visual inspection of the model suggests that the impact of latency becomes especially noticeable starting from the $250 \mathrm{~ms}$ mark. Furthermore, the largest impact of latency - that is, the latency value where the change in reaction time is the steepest-occurs at $407 \mathrm{~ms}$. This can be determined by calculating the inflection point of the polynomial: $\frac{-\beta_{2}}{3 \times \beta_{3}}=407 \mathrm{~ms}$.

To investigate block-wise differences in the impact of latency on reaction times, we conducted an additional oneway repeated-measures ANOVA on the median reaction times. Our predictor variable's levels were labeled with the expected value of the added latency for respective blocks, increased with the base latency. For instance, in the block where the added latency was taken from a uniform distribution ranging between 0 and $83.33 \mathrm{~ms}$, the expected value for the added latency $(\mathrm{E}($ latency) $)$ is $41.7 \mathrm{~ms}$. Adding the base latency to this number gives us approximately $162 \mathrm{~ms}$. The six blocks were characterized by the following levels of $\mathrm{E}$ (latency): 162, 245, 328, 412, 495, and $578 \mathrm{~ms}$, for blocks A through F (see fig. 2 and table II).

Mauchly's test indicated that the assumption of sphericity had been violated $(W=0.334, p=.001)$, therefore degrees of freedom were corrected using Greenhouse-Geisser estimates of sphericity $(\epsilon=701)$. The effect of latency was significant
$((F(5,180=12.901, p<0.001)$. To identify which adjacent factor levels differed significantly, a follow-up set of pairwise t-tests was conducted. The latency increase between adjacent latency levels $\mathrm{E}($ latency $)=328 \mathrm{~ms}$ and $\mathrm{E}($ latency $)=$ $412 \mathrm{~ms}$ yielded the only significant effect on median reaction times. Note that this coincides with the inflection point-i.e., the steepest effect of latency on reaction time-found in the quantile regression curve (407 ms). However, the effect did not survive Holm's correction for multiple comparisons [57].

TABLE I

QUANTILE REGRESSION MODELLING STEPS.

\begin{tabular}{ccccc}
\hline & 1st order & 2nd order & 3rd order & 4th order \\
\hline (Intercept) & $\mathbf{6 . 2 4 e 2} * * *$ & $\mathbf{7 . 9 9 e 2} * *$ & $\mathbf{1 . 2 9 e 3} * * *$ & $6.99 \mathrm{e} 2$ \\
& $(2.24 \mathrm{e} 1)$ & $(6.28 \mathrm{e} 1)$ & $(1.59 \mathrm{e} 2)$ & $(3.95 \mathrm{e} 2)$ \\
latency & $\mathbf{1 . 1 3} * * *$ & $-8.75 \mathrm{e}-2$ & $\mathbf{- 5 . 1 1} * *$ & 3.10 \\
& $(0.072)$ & $(0.411)$ & $(1.614)$ & $(5.480)$ \\
latency $^{2}$ & & $\mathbf{1 . 7 6 e - 3} * *$ & $\mathbf{1 . 6 9 e - 2} * * *$ & $-2.21 \mathrm{e}-2$ \\
& & $(6.0 \mathrm{e}-4)$ & $(4.97 \mathrm{e}-3)$ & $(-2.21 \mathrm{e}-2)$ \\
latency $^{3}$ & & & $\mathbf{- 1 . 0 0 e - 5 * *}$ & $6.00 \mathrm{e}-5$ \\
& & & $(0.0)$ & $(0.50 \mathrm{e}-4)$ \\
latency $^{4}$ & & & & $-5.19 \mathrm{e}-8$ \\
& & & & $(0.0)$ \\
\hline$F$ & & & & 2.027 \\
$\mathrm{AIC}$ & 139161.8 & 139151.1 & 139140.9 & 139139.6
\end{tabular}

Coefficients and standard errors (in parentheses) are reported per model, as well as the corresponding AIC score. F-test statistics represent a comparison with the previous best model, showing an optimal fit for the 3rd order model. ***p $<0.001$, **p $<0.01$, $\mathrm{p}<0.05$

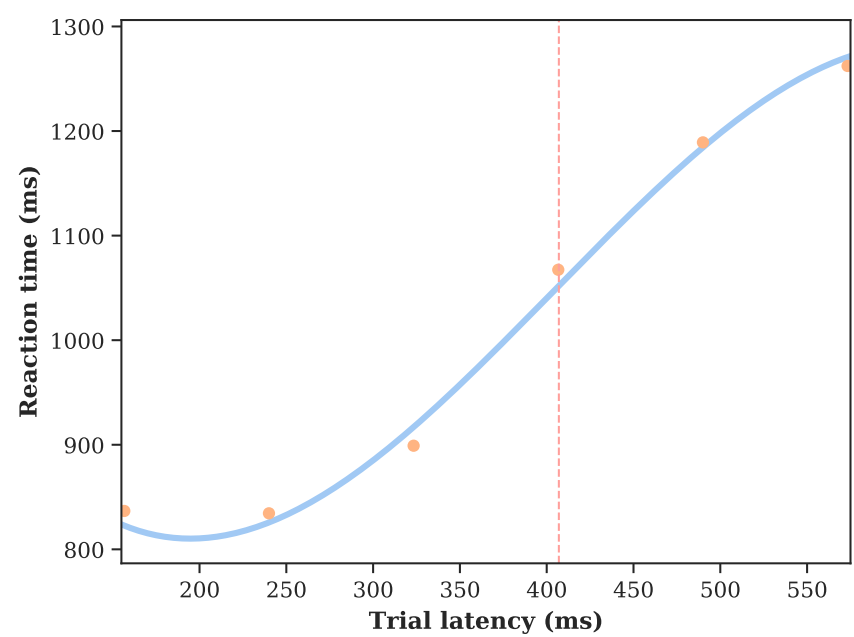

Fig. 4. Polynomial quantile regression fit of reaction time on trial latency: dots - median reaction time per condition; solid - polynomial fit; dashed latency value corresponding with inflection point (407 ms).

TABLE II

REACTION TIME STATISTICS (MS)

\begin{tabular}{ccccc}
\hline Block & E(latency) & mean & median & sd \\
\hline A & 162 & 1055.9 & 836.702 & 673.119 \\
B & 245 & 1101.26 & 834.452 & 786.332 \\
C & 328 & 1226.828 & 899.085 & 881.244 \\
D & 412 & 1476.922 & 1067.341 & 1205.301 \\
E & 495 & 1669.119 & 1189.166 & 1339.656 \\
F & 578 & 1844.26 & 1262.214 & 1534.518 \\
\hline
\end{tabular}




\section{B. Quality of Experience}

We explored the impact of latency on the player's Quality of Experience by exploring the post-block survey data. Since these variables were only queried after each block, the predictor variable levels were again labeled with the expected value of the latency for each respective block.

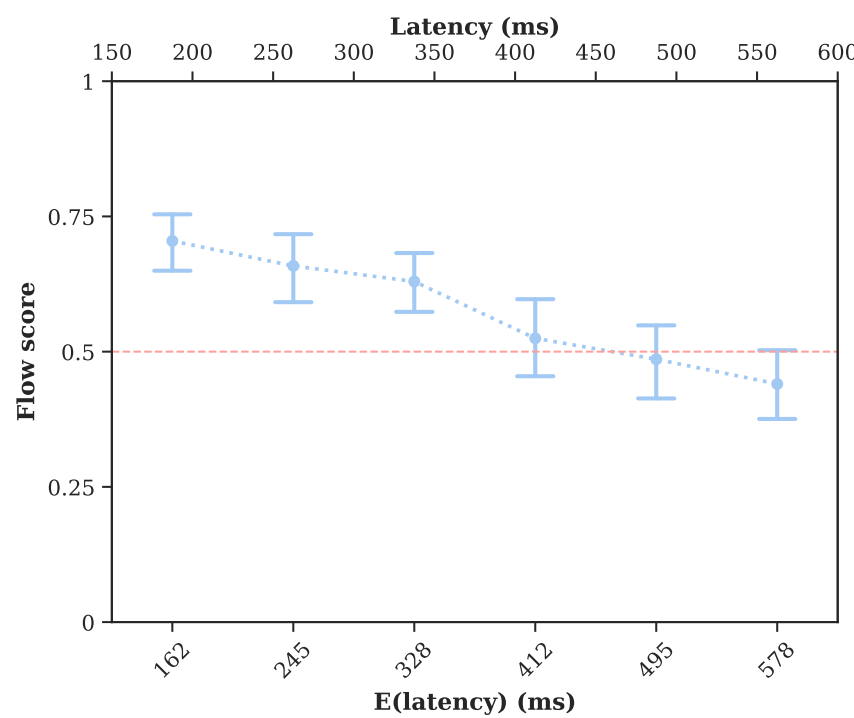

Fig. 5. Mean flow ratings per condition (normalized). Whiskers - $95 \%$ confidence intervals; dashed horizontal - neutral flow rating.

First, we examined the effect of increased latency on the flow scores (fig. 5). We did so using a one-way repeated-measures analysis of variance (ANOVA). The effect of latency was significant $(F(5,180)=26.83, p<0.001)$. Adjacent factor levels were then compared using a follow-up set of pair-wise ttests, while controlling for multiple testing [57]. All conditions differed significantly after correction (all $p_{\text {corrected }}<0.05$, barring immediately adjacent ones. A single exception to this pattern is the significant decrease in flow between the adjacent 328 and 412 ms conditions $(t(36)=3.28, p=0.02)$.

Next, we analyzed ratings for latency awareness, sense of adaptability, sense of efficiency, and disturbance, using repeated-measures ANOVAs. For the sense of efficiency and adaptability, Mauchly's test indicated that the assumption of sphericity had been violated (both $W=0.48, p=.03$ ), therefore degrees of freedom were corrected using GreenhouseGeisser estimates of sphericity $(\epsilon=0.78$ and $\epsilon=0 ; 80$, respectively). All ratings were significantly affected by the latency manipulation: latency awareness and disturbance increased with latency $\left(F(5,180)=55.81, p<.001, \eta^{2}=0.45\right.$ and $F(5,180)=34.56, p<.001, \eta^{2}=0.34$, respectively), whereas efficiency and adaptability decreased with latency $\left(F(3.9,140.52)=22.14, p<.001, \eta^{2}=0.28\right.$ and $F(3.99,143.78)=10.15, p<.001, \eta^{2}=0.15$ respectively $)$. Interestingly, each of these ratings followed a similar, quasilinear pattern, crossing the neutral point (fig. 6) from the 412 ms condition on.

Finally, we analyzed the sum scores for the IMI subscales interest and enjoyment and perceived competence,
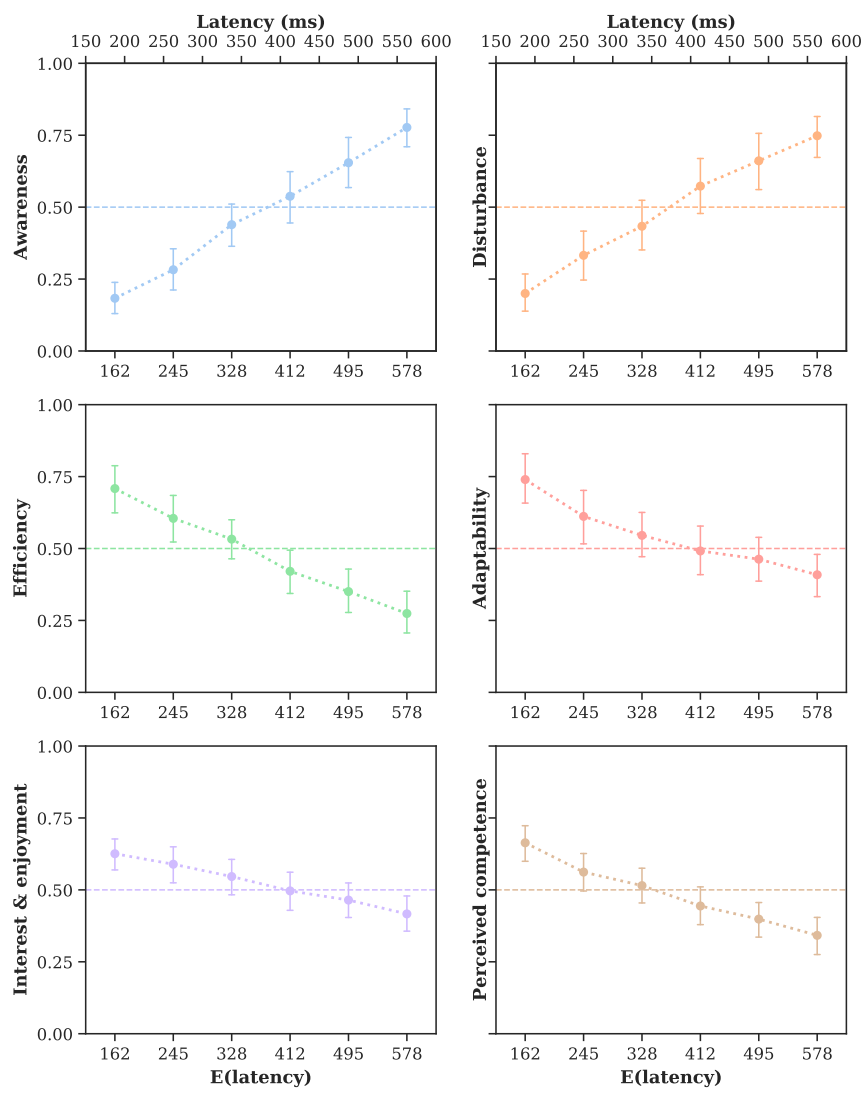

Fig. 6. Mean ratings per condition for adaptability, awareness, efficiency, disturbance, interest \& enjoyment (IMI), and perceived competence (IMI), (all ratings normalized). Whiskers - $95 \%$ confidence intervals; dashed horizontal neutral rating.

using repeated-measures ANOVAs. For perceived competence, Mauchly's test indicated that the assumption of sphericity had been violated $(W=0.50, p=.05)$, therefore degrees of freedom were corrected using Greenhouse-Geisser estimates of sphericity $(\epsilon=0.80)$. Ratings on both scales were significantly impacted by our latency manipulation (interest and enjoyment: $F(5,180)=16.22, p<.001, \eta^{2}=0.12$; perceived competence: $\left.F(4,144.1)=28.6, p<.001, \eta^{2}=0.22\right)$, showing a monotonous decrease in both cases. Again, mean scores for both ratings drop below the neutral point from 412 ms onwards (fig. 6).

\section{DISCUSSION}

Technological advancement has helped bring Internet network latencies down, while simultaneously enabling richer application platforms that use wireless networks and have more computational demands, potentially increasing local latency. Exergames-where players engage with a game through active movement—are particularly at risk, as they often depend on more complex peripherals to accurately register player movement. The aim of our study was to determine acceptable local latencies in the context of exergaming, and where the threshold lies beyond which player experience and performance begin to suffer. We conducted a 37-person user study where participants played a custom-made desktop-based 
exergame which introduced artificial latency and recorded player performance (reaction time) and various measures of the player's user experience (e.g., flow).

As expected, an increase in latency causes player performance to deteriorate. More specifically, performance decrease follows an S-shaped curve. At low values (under $200 \mathrm{~ms}$ ), latency has a minimal impact on performance, but the latency impact increases for moderate values (300-500 ms), and begins to cap at high values (above $500 \mathrm{~ms}$ ). The tipping point-i.e., the point where latency appears to have to most severe impactis at about $400 \mathrm{~ms}$. Similarly, latency has a negative impact on the players' experience. The most significant decrease in flow is at about $350 \mathrm{~ms}$. From that point, mean flow ratings approached the neutral point. Players do not experience a heightened sense of pleasant engagement with the game and its mechanisms[25], nor do they completely reject the experience: they are simply indifferent. This pattern is reflected in almost all of the other ratings. With increasing degrees of latency, players become more aware and feel more disturbed by the delays, begin to feel less efficient in their actions, and cannot adapt to the delay as well. For each of these ratings, the neutral line is crossed with latency values of about $350 \mathrm{~ms}$. Where players would first disagree with the items (e.g., 'Indicate how disturbing you found the latency'), they now pivot to positive responses-or vice versa. Correspondingly, the same trend can be observed in the subscales of the Intrinsic Motivation Inventory [52]. With increasing degrees of latency, players report less interest and enjoyment, and begin to feel less competent. The neutral threshold is again crossed at the same 350-millisecond mark.

Relative to the spectrum of game genres, our results suggest that single player, local exergames are less susceptible to latency than first-person shooters, for which latencies of $100 \mathrm{~ms}$ or less have been shown to hamper performance both in local $[9,58]$ and networked play sessions [12,59]. Real-time strategy games, in turn, have been associated with noticeably higher latency thresholds. A study by Claypool [14] suggested that latencies under 1 second, while perceptible, were easy to adapt to from a player's perspective, and player performance was largely unaffected by seconds of latency. The authors attribute this sizeable latency tolerance to the very nature of the games, which "emphasize strategy more than the interactive aspects" [14, p.68] The opposite is true for our exergame, in which quick reflexes are the player's primary assets.

The effects of latency on our exergame are perhaps most similar to games with a third-person perspective, where players see and 'puppeteer' a game character. In these games, performances begin to degrade when latency reaches hundreds of $\mathrm{ms}[32,60]$. This latency tolerance similarity may be at least partially explained by the indirect nature of the interaction for both our exergame and third-person perspective games-in both cases, players indirectly control an avatar (a silhouette, in our exergame) that in turn interacts with the game world. Note that lower latency thresholds have been proposed for third-person games [61], although such stricter criteria can likely be attributed to the multi-user nature of the games that were studied. In massive multiplayer online role-playing games, for instance [13], the most important source of latency is the player's connectivity to the game server. In this case, latency has a different contextual meaning, as it can hinder interactions with other players, or bring about competitive disadvantages. In contrast, our study zoomed in on local latency in a single player exergame setting.

At present, a new generation of games and experiences is still fighting its way to the mainstream: extended (virtual, augmented or mixed) reality applications. Recent studies suggest that latency thresholds are more stringent when users are placed in virtual reality. First, end-to-end latency values over $58 \mathrm{~ms}$ can already lead users to experience cybersickness - a feeling of nausea triggered by visual vestibular mismatch [38]. This is not typically a concern for desktop-based exergames, as the frame of reference (i.e., the room in which players find themselves) is still fundamentally real from a sensory perspective. Second, one of the oft-used principal markers for a 'good' virtual reality experience is the ability to convince users they are present [62] in the alternate reality. There is research to suggest that the illusion of embodiment, at least, suffers from latency values upwards of $192 \mathrm{~ms}$, though overall measures of agency and presence appear more resilient in that respect [38, 63]. Third and finally, performance in virtual reality settings can already be impacted by relatively small amounts of delay (e.g., 69 ms [38]), which in turn can be expected to be of gradually increasing detriment to the user experience.

Using a custom-made exergame imposed some limitations. Depending on the configuration of the game parameters, the impact of latency can be expected to shift patterns here presented. For instance, our game used moving targets which moved with speeds selected at random in the interval between 0.128 and $0.642 \mathrm{~m} / \mathrm{s}$. These boundaries were set during the piloting phase, where no artificial latency was added, in an effort to create diverse and sufficiently-but not overlychallenging gameplay. Naturally, it is possible to impair both performances and user experiences by, for instance, increasing the target speed, making the game significantly more difficult.

Second, our exergame was rudimentary in many respects. Little attention was devoted to the visual aspects of the game: no specific graphics are used apart from the user's silhouette, the targets, the green zone that helped players to reset their position, and some basic score information. The game's auditory feedback was simple, to the point of it being perceived as monotonous by some participants. More fleshedout games may be more intrinsically captivating, potentially affecting the player's tolerance for latency.

Third, the game was played offline without any networking to a server or other players. While the presence of scoring information can be expected to spark indirect competitiveness in some players, at no point did they directly compete with each other. As mentioned earlier, game settings in which players can directly cooperate or compete can conceivably lower the amount of latency a player is willing to bear. However, results from a recent study support our findings [64]. In this experiment, which made use of a multiplayer virtual reality (VR) exergame, various degrees of artificial network latency were introduced, either on the side of the user, or the side of the (artificial) opponent. Interestingly, the quality of experience was only affected when the latency on the user-side was high (500 ms). The latency on the side of the opponent, in turn, did not appear 
to influence the player's experience. This pattern was also visible in flow ratings, which only significantly dropped when the user's network latency was high.

Fourth, the participants in our experiment were all healthy volunteers of a fairly young age (28 years old, on average). Exergames are often used to improve motor skills, or prevent their deterioration, in older adults [65] and can also function as a therapeutic tool, facilitating physical rehabilitation programs $[10,66]$. We must be cautious in generalizing our present findings to elderly or clinical populations without additional testing. For instance, the impact of latency can be expected to be more pronounced with less mobile individuals, whose reaction time may be slower in general.

Designing a perfectly archetypical exergame is challenging. Still, we argue that our specific exergame instance offers a good perspective on how latency impacts a player's experience, as well as a valuable approximation for the critical tipping point that should be observed by developers. Future work could broaden these results in several ways. For instance, it could further explore the potential confound between the added latency in a trial and the speed with which the target moved. Follow-up studies could also explore the role of the input device-in our case: the player's body-by juxtaposing our exergame with a comparable mouse-and-keyboard version. Finally, it may be interesting to investigate the impact of the medium by juxtaposing our desktop application with an VR-based variant (e.g., by exploring a VR version of Spaz!). More specifically, it is unclear to what extent the nature of the experience-i.e., exergaming - is responsible for the high latency tolerances reported by users, and how these findings extrapolate to fully immersive experiences.

\section{CONCLUSION}

Results from our 37-person user study show an increase in local latency causes player performance to deteriorate following an S-shaped curve-latencies under $300 \mathrm{~ms}$ minimally impact performance, latencies between 300 and 500 ms sharply degrade performance, and latencies above $500 \mathrm{~ms}$ have an impact plateau. Similarly, latency degrades player quality of experience, most sharply around $350 \mathrm{~ms}$ where positive player flow drops by about $50 \%$. These bounds suggest a latency target budget of no more than $300 \mathrm{~ms}$ total for exergame developers when developing for desktop-based platforms. These effects are relatively forgiving compared to traditional first-person computer games that are severely affected by latencies under $150 \mathrm{~ms}[12,59]$, but are akin to some latency tolerances for third-person computer games $[32,60]$ and less than latencies for some omni-present computer games [14].

\section{ACKNOWLEDGMENT}

The exergame used in this study was made by Gaël Vanhalst and Michiel Houwen at Digital Arts and Entertainment Research, Howest, Belgium. This research is funded by the imec.icon project iPlay HBC.2016.0656, which was cofinanced by imec and VLAIO and brings together the following partners: Barco, RSscan, RSE, Howest, DAE Studios, imec-IPI-UGent, imec-mict-UGent, and FaBeR.

\section{REFERENCES}

[1] B. Ferguson, "The Emergence of Games for Health," Games for Health Journal, vol. 1, no. 1, 2012.

[2] X. Wang and A. Perry, "Metabolic and Physiologic Responses to Video Game Play in 7- to 10-Year-Old Boys," Pediatrics \& Adolescent Medicine, vol. 160, no. 4, 2006.

[3] R. Kretschmann, "Exergames and Health Promotion Nintendo Wii Sports: Physiological Measures vs. Perceived Opinions," in Proceedings of the VII Congress of the International Association of Colleges of Physical Education, 2010.

[4] S. Siegel, B. Haddock, A. Dubois, and L. Wilkin, "Active Video/Arcade Games (Exergaming) and Energy Expenditure in College Students," Exercise Science, vol. 2, no. 3, 2009.

[5] Postive Gaming, "The Benefits of Exergaming," Sep. 2019.

[6] M. Claypool and K. Claypool, "Latency can kill: precision and deadline in online games," Proceedings of the first annual ACM Multimedia Systems Conference, pp. 215-222, 2010. [Online]. Available: http://dl.acm.org/ citation.cfm?id=1730863

[7] K.-T. Chen, P. Huang, and C.-L. Lei, "How sensitive are online gamers to network quality?" Communications of the ACM, vol. 49, no. 11, p. 34, 2007.

[8] J. Nielsen, "Nielsens Law of Internet Bandwidth," 2018. [Online]. Available: https://www.nngroup.com/articles/ law-of-bandwidth/

[9] Z. Ivkovic, I. Stavness, C. Gutwin, and S. Sutcliffe, "Quantifying and Mitigating the Negative Effects of Local Latencies on Aiming in 3D Shooter Games," Proceedings of the 33rd Annual ACM Conference on Human Factors in Computing Systems, pp. 135-144, 2015.

[10] K. Tanaka, J. Parker, G. Baradoy, D. Sheehan, J. R. Holash, and L. Katz, "A Comparison of Exergaming Interfaces for Use in Rehabilitation Programs and Research," Loading... The Journal of the Canadian Game Studies Association, vol. 6, no. 9, pp. 69-81, 2012.

[11] R. Amin, F. Jackson, J. E. Gilbert, J. Martin, and T. Shaw, "Assessing the Impact of Latency and Jitter on the Perceived Quality of Call of Duty Modern Warfare 2," in Proceedings of HCI - Users and Contexts of Use. Las Vegas, NV, USA: Springer-Verlag, Jul. 2013, pp. 97-106.

[12] G. Armitage, "An Experimental Estimation of Latency Sensitivity in Multiplayer Quake 3," in Proceedings of the 11th IEEE International Conference on Networks (ICON), Sydney, Australia, Sep. 2003.

[13] K. Chen, P. Haung, G. Wang, C. Huang, and C. Lee, "On the Sensitivity of Online Game Playing Time to Network QoS," in Proceedings of IEEE Infocom, Barcelona, Spain, Apr. 2006.

[14] M. Claypool, "The effect of latency on user performance in Real-Time Strategy games," Computer Networks, vol. 49, no. 1, pp. 52-70, 2005.

[15] T. Beigbeder, R. Coughlan, C. Lusher, J. Plunkett, E. Agu, 
and M. Claypool, "The Effects of Loss and Latency on User Performance in Unreal Tournament 2003," in Proceedings of ACM Network and System Support for Games Workshop (NetGames), Portland, OG, USA, Sep. 2004.

[16] M. Long and C. Gutwin, "Characterizing and Modeling the Effects of Local Latency on Game Performance and Experience," in Proceedings of the ACM Symposium on Computer-Human Interaction in Play (CHI Play), Melbourne, VC, Australia, 2018.

[17] M. Claypool, R. Eg, and K. Raaen, "Modeling User Performance for Moving Target Selection with a Delayed Mouse," in Proceedings of the 23rd International Conference on MultiMedia Modeling (MMM), Reykjavik, Iceland, Jan. 2017.

[18] A. A. Hajri, S. Fels, G. Miller, and M. Ilich, "Moving Target Selection in 2D Graphical User Interfaces," in Proceeding of IFIP TC Human-Computer Interaction (INTERACT), Lisbon, Portugal, Sep. 2011.

[19] I. S. MacKenzie and W. Buxton, "Extending Fitts' Law to Two-Dimensional Tasks," in Proceedings of the ACM CHI Conference on Human Factors in Computing Systems, Monterey, CA, USA, May 1992, pp. 219 - 226.

[20] R. Jota, A. Ng, P. Dietz, and D. Wigdor, "How Fast is Fast Enough?: A Study of the Effects of Latency in Direct-touch Pointing Tasks," in Proceedings of the ACM SIGCHI Conference on Human Factors in Computing Systems, Paris, France, 2013.

[21] K. Raaen and R. Eg, "Instantaneous Human-Computer Interactions: Button Causes and Screen Effects," in Proceedings of the 17th HCI International Conference, Los Angeles, CA, USA, Aug. 2015.

[22] A. Pavlovych and W. Stuerzlinger, "Target Following Performance in the Presence of Latency, Jitter, and Signal Dropouts," in Proceedings of Graphics Interface, St. John's, NL, Canada, May 2011, pp. 33-40.

[23] M. Long and C. Gutwin, "Effects of local latency on game pointing devices and game pointing tasks," in Proceedings of the ACM SIGCHI Conference on Human Factors in Computing Systems (CHI), Glasgow, Scotland, UK, may 2019.

[24] D. J. Palidis, P. A. Wyder-Hodge, J. Fooken, and M. Spering, "Distinct eye movement patterns enhance dynamic visual acuity," PLoS ONE, vol. 12, no. 2, pp. 1-14, 2017.

[25] M. Csikszentmihalyi, "Play and Intrinsic Rewards," Journal of Humanistic Psychology, vol. 15, pp. 41-63, 1975.

[26] G. B. Moneta and M. Csikszentmihalyi, "The Effect of Perceived Challenges and Skills on the Quality of Subjective Experience," Journal of Personality, vol. 64, pp. 274-310, 1996.

[27] K. Procci, A. R. Singer, K. R. Levy, and C. Bowers, "Measuring the flow experience of gamers: An evaluation of the DFS-2," Computers in Human Behavior, vol. 28, no. 6, pp. 2306-2312, 2012. [Online]. Available: http://dx.doi.org/10.1016/j.chb.2012.06.039

[28] S. Arnab, A. Perttula, and M. Suominen, "Flow experience as a quality measure in evaluating physically activating serious games," Lecture Notes in Computer Science (in- cluding subseries Lecture Notes in Artificial Intelligence and Lecture Notes in Bioinformatics), vol. 8605, pp. 200212, 2014.

[29] A. Koivisto, S. Merilampi, and K. Kiili, "Mobile Exergames for Preventing Diseases Related to Childhood Obesity," in Proceedings of the 4th International Symposium on Applied Sciences in Biomedical and Communication Technologies (ISABEL), Barcelona, Spain, 2011.

[30] M. Claypool and K. Claypool, "Latency and player actions in online games," Communications of the ACM, vol. 49, no. 11, p. 40-45, Nov. 2006. [Online]. Available: https://doi.org/10.1145/1167838.1167860

[31] L. Pantel and L. C. Wolf, "On the Impact of Delay on Real-Time Multiplayer Games," in Proceedings of the Workshop on Network and Operating Systems Support for Digital Audio and Video (NOSSDAV), Miami, FL, USA, May 2002.

[32] T. Fritsch, H. Ritter, and J. H. Schiller, "The Effect of Latency and Network Limitations on MMORPGs: a Field Study of Everquest 2," in Proceedings of the 4th ACM Network and System Support for Games (NetGames), Hawthorne, NY, USA, Oct. 2005.

[33] Y. W. Bernier, "Latency Compensating Methods in Client/Server In-game Protocol Design and Optimization," in Proceedings of the Game Developers Conference, San Francisco, CA, USA, Feb. 2001.

[34] P. M. Fitts, "The Information Capacity of the Human Motor System in Controlling the Amplitude of Movement," Journal of Experimental Psychology, vol. 47, no. 6, pp. 381-391, Jun. 1954.

[35] M. Claypool, "Game Input with Delay - Moving Target Selection with a Game Controller Thumbstick," ACM Transactions on Multimedia Computing, Communications, and Applications (TOMM) - Special Section on DelaySensitive Video Computing in the Cloud, vol. 14, no. 3s, Aug. 2018.

[36] K. Raaen, R. Eg, , and I. Kjellmo, "Playing with Delay: an Interactive VR Demonstration," in Proceedings of the 11th ACM Workshop on Immersive Mixed and Virtual Environment Systems (MMVE), Amherst, MA, USA, 2019.

[37] T. Waltemate, I. Senna, F. Hülsmann, M. Rohde, S. Kopp, M. Ernst, and M. Botsch, "The Impact of Latency on Perceptual Judgments and Motor Performance in ClosedLoop Interaction in Virtual Reality," in Proceedings of the 22nd ACM Conference on Virtual Reality Software and Technology (VRST). Munich, Germany: Association for Computing Machinery, Nov. 2016, p. 27-35. [Online]. Available: https://doi.org/10.1145/2993369.2993381

[38] G. S. Caserman P., Martinussen M., "Effects of Endto-end Latency on User Experience and Performance in Immersive Virtual Reality Applications," in Proceedings of the IFIP Entertainment Computing and Serious Games, Arequipa, Peru, Nov. 2019.

[39] P. Sweetser and P. Wyeth, "GameFlow: A Model for Evaluating Player Enjoyment in Games," Computers in Entertainment, vol. 3, no. 3, pp. 1-24, 2005.

[40] D. M. Bressler and A. M. Bodzin, "A mixed methods assessment of students' flow experiences during a mobile 
augmented reality science game," Journal of Computer Assisted Learning, 2013.

[41] J. Sinclair, P. Hingston, and M. Masek, "Exergame development using the dual flow model," in Proceedings of the 6th Australasian Conference on Interactive Entertainment, IE 2009, 2009.

[42] A. Bodzin, R. A. Junior, T. Hammond, and D. Anastasio, "Investigating Engagement and Flow with a Placed-Based Immersive Virtual Reality Game," Journal of Science Education and Technology, vol. 30, no. 3, pp. 347360, 2021. [Online]. Available: https://doi.org/10.1007/ s10956-020-09870-4

[43] J. Young and M. Pain, "The Zone: Evidence of a universal phenomenon for athletes across sports," Athletic Insight : the online journal of sport psychology, vol. 1, no. 3, pp. 21-30, 1999.

[44] M. Csikszentmihalyi, Flow: The Psychology of Optimal Experience. New York, NY: Harper Perennial, 1990.

[45] B. Berberian, P. Le Blaye, C. Schulte, N. Kinani, and P. R. Sim, "Data transmission latency and sense of control," in Engineering Psychology and Cognitive Ergonomics. Understanding Human Cognition, D. Harris, Ed. Berlin, Heidelberg: Springer Berlin Heidelberg, 2013, pp. 3-12.

[46] M. Booth, "Assessment of physical activity: An international perspective," Research Quarterly for Exercise and Sport, 2000.

[47] A. Kumcu, L. Vermeulen, S. A. Elprama, P. Duysburgh, L. Platisa, Y. Van Nieuwenhove, N. Van De Winkel, A. Jacobs, J. Van Looy, and W. Philips, "Effect of video lag on laparoscopic surgery: correlation between performance and usability at low latencies," The international journal of medical robotics + computer assisted surgery : MRCAS, vol. 7, no. May, pp. 375-392, 2011.

[48] S. Jamieson, "Likert scales: How to (ab)use them," Medical Education, vol. 38, no. 12, pp. 1217-1218, 2004.

[49] F. Funke and U. D. Reips, "Why Semantic Differentials in Web-Based Research Should Be Made from Visual Analogue Scales and Not from 5-Point Scales," Field Methods, vol. 24, no. 3, pp. 310-327, 2012.

[50] J. Hofmans and P. Theuns, "On the linearity of predefined and self-anchoring Visual Analogue Scales," British Journal of Mathematical and Statistical Psychology, vol. 61, no. 2, pp. 401-413, 2008.

[51] S. Engeser and F. Rheinberg, "Flow, performance and moderators of challenge-skill balance," Motivation and Emotion, vol. 32, no. 3, pp. 158-172, 2008.

[52] E. L. Deci and R. M. Ryan, "Self-Determination Theory and the Facilitation of Intrinsic Motivation, Social Development, and Well-Being," American Psychologist, vol. 55, no. 1, pp. 68-78, 2000.

[53] K. Raaen and A. Petlund, "How Much Delay is there Really in Current Games?" in Proceedings of the 6th ACM Multimedia Systems Conference (MMSys), Portland, OR, USA, Mar. 2015.

[54] G. Casiez, S. Conversy, M. Falce, S. Huot, and N. Roussel, "'looking through the eye of the mouse: A simple method for measuring end-to-end latency using an optical mouse"," in Proceedings of the 28th Annual ACM Symposium on
User Interface Software \& Technology (UIST), Daegu, Kyungpook, Republic of Korea, 2015.

[55] K. Raaen and I. Kjellmo, "Measuring Latency in Virtual Reality Systems," in Proceedings of International Conference on Entertainment Computing (ICEC), Trondheim, Norway, Sep. 2015.

[56] R. Koenker and K. F. Hallock, "Quantile Regression: An Introduction," 2000. [Online]. Available: http: //www.econ.uiuc.edu/ $\{\sim\}$ roger/research/intro/rq3.pdf

[57] S. Holm, "A simple sequentially rejective multiple test procedure," Scandinavian Journal of Statistics, vol. 6, no. 2, pp. 65-70, 1979.

[58] S. Liu, A. Kuwahara, J. Sherman, J. Scovell, and M. Claypool, "Lower is Better? The Effects of Local Latencies on Competitive First Person Shooter Game Players," in Proceedings of theACM Conference on Human Factors in Computing Systems (CHI), Virtual Conference, May 8-13 2021.

[59] S. Liu, A. Kuwahara, J. Scovell, J. Sherman, and M. Claypool, "The Effects of Network Latency on Competitive First-Person Shooter Game Players," in Proceedings of the 13th International Conference on Quality of Multimedia Experience (QoMEX), Virtual Conference, Jun. 2021.

[60] M. Claypool and K. Claypool, "Latency and player actions in online games," Communications of the ACM, vol. 49, no. 11, p. 40, Nov. 2006.

[61] K. Raaen and T.-M. Grønli, "Latency Thresholds for Usability in Games: A Survey," in Norsk Informatikkonferanse, 2014.

[62] J. De Letter, A. Zheleva, M. Maes, A. All, L. De Marez, and W. Durnez, "What did you expect?" Quality and User Experience, vol. 6, no. 1, pp. 1-13, 2021. [Online]. Available: https://doi.org/10.1007/s41233-021-00045-6

[63] T. Waltemate, I. Senna, F. Hülsmann, M. Rohde, S. Kopp, M. Ernst, and M. Botsch, "The impact of latency on perceptual judgments and motor performance in closedloop interaction in virtual reality," Proceedings of the ACM Symposium on Virtual Reality Software and Technology, VRST, vol. 02-04-Nove, pp. 27-35, 2016.

[64] T. Kojic, S. Schmidt, S. Moller, and J. N. Voigt-Antons, "Influence of network delay in virtual reality multiplayer exergames: Who is actually delayed?" 2019 11th International Conference on Quality of Multimedia Experience, QoMEX 2019, 2019.

[65] T. B. F. Pacheco, C. S. P. de Medeiros, V. H. B. de Oliveira, E. R. Vieira, and F. A. d. C. Cavalcanti, "Effectiveness of exergames for improving mobility and balance in older adults: A systematic review and meta-analysis." Systematic Reviews, 2020.

[66] M. Pirovano, E. Surer, R. Mainetti, P. L. Lanzi, and N. Alberto Borghese, "Exergaming and rehabilitation: A methodology for the design of effective and safe therapeutic exergames," Entertainment Computing, vol. 14, pp. 55-65, 2016. [Online]. Available: http: //dx.doi.org/10.1016/j.entcom.2015.10.002 